This item was submitted to Loughborough's Research Repository by the author.

Items in Figshare are protected by copyright, with all rights reserved, unless otherwise indicated.

\title{
Gold adatoms and dimers on relaxed graphite surfaces
}

PLEASE CITE THE PUBLISHED VERSION

PUBLISHER

(C) American Physical Society

LICENCE

CC BY-NC-ND 4.0

\section{REPOSITORY RECORD}

Wang, G.M., J.J. BelBruno, Steven D. Kenny, and Roger Smith. 2019. "Gold Adatoms and Dimers on Relaxed Graphite Surfaces". figshare. https://hdl.handle.net/2134/1746. 


\title{
Gold adatoms and dimers on relaxed graphite surfaces
}

\author{
Guan Ming Wang, ${ }^{1}$ Joseph J. BelBruno, ${ }^{1}$ Steven D. Kenny, ${ }^{2}$ and Roger Smith ${ }^{2}$ \\ ${ }^{1}$ Center for Nanomaterials Research and Department of Chemistry, Dartmouth College, Hanover, New Hampshire 03755, USA \\ ${ }^{2}$ Department of Mathematical Sciences, Loughborough University, Loughborough, Leicestershire, LE11 3TU, United Kingdom
}

(Received 15 August 2003; revised manuscript received 8 January 2004; published 21 May 2004)

\begin{abstract}
The interaction of deposited gold adatoms and dimers with multilayer relaxed graphite surfaces is investigated through a density functional approach with numerical orbitals and a relativistic core pseudopotential. The energy landscape for a gold adatom along [110] agrees with scanning tunneling microscopy observations including the preferred $\beta$ binding site for adatoms and the mobility difference between silver and gold adatoms. Deposited particles are shown to induce surface deformation and polarization. Static relaxation and dynamic simulations indicate that the energetically preferred binding orientation for a gold dimer is normal rather than parallel to the graphite surface. The dimer response to a simulated scanning tunneling microscopy tip is investigated by molecular dynamics simulations.
\end{abstract}

DOI: 10.1103/PhysRevB.69.195412

PACS number(s): 79.20.Rf, 82.20.Wt, 36.40.Sx

\section{INTRODUCTION}

The computational study of clusters on surfaces has been an important asset in understanding surface phenomena. ${ }^{1-5}$ Most commonly, model potentials are used to simulate experimental observations. More accurate, but computationally expensive $a b$ initio approaches ${ }^{6,7}$ have been attempted on a relatively small scale, especially for the gold-graphite system of interest here. The cluster-surface system consists of two distinct parts and both, clusters and surfaces, have extensive computational histories. Many well-known ab initio methods have been applied to atomic clusters, especially those of gold. ${ }^{8-11}$ For surfaces, periodic boundary conditions can be used with minimal-sized computational cells to achieve accurate results. Several approaches have been applied to cluster-surface systems: the 'cluster, ${ }^{6}$ supercell and model potential schemes. ${ }^{7,12}$ Cluster techniques substitute an appropriate molecule for a portion of the surface. The cluster size must be sufficiently large so as to avoid "edge effects" caused by the coordinately unsaturated edge atoms. The supercell technique employs periodic boundary conditions with a fixed cell. While edge effects are eliminated by this method, the supercell must be sufficiently large so as to eliminate the coupling between adsorbates on adjacent cells. Potential model simulations of cluster-surface phenomena ${ }^{13-15}$ use a large cluster model and provide good results. For example, the Morse potential has been widely used for cluster-surface interactions. ${ }^{13,16,17}$ However, this type of simulation often encounters difficulties dealing with small clusters, charge transfer situations and induced surface charges.

There were several previous computational studies of the gold/graphite or related systems. Duffy and Blackman ${ }^{6}$ calculated structures for silver adatoms and dimers on two fixed layers of graphite. Moullet ${ }^{7}$ completed $a b$ initio calculations for aluminum clusters on a graphite surface with a single relaxed layer. A single surface layer cannot differentiate between $\alpha$ (carbon atom binding sites for which the surface atom has a carbon atom directly below it in the next layer) and $\beta$ (carbon atom binding sites for which the surface atom does not have a carbon atom directly below it in the next layer) sites on the surfaces. ${ }^{18}$ In our related research with silver adatoms and dimers, ${ }^{12}$ relaxation of the graphite surface was found to affect the preferential order of the binding sites. It was also noted that additional layers of graphite play a role in determining the favored binding site for a single silver atom.

Experimentally, adatoms and dimers of silver and gold were imaged by scanning tunneling microscopy (STM) techniques in the late 1980s. ${ }^{19}$ These data may be used for qualitative comparisons with our computational results. The STM image of a gold dimer, apparently oriented parallel to the surface, was observed to span a distance somewhat longer than that expected for an isolated (nonsurface bound) dimer. To the best of our knowledge, there are no other images in the literature and no connection between experiment and computation has been made for these particular images.

In this paper, we report a wide range of related results, beginning with structural results for gold adatoms on four layers of graphite. The package for linear atomic-type orbitals (PLATO) (Ref. 20) that we employed implements a density functional method with the local density approximation and optimized orbitals from neutral atoms and positively charged ions. Secondly, we report our studies for the static relaxation of possible parallel and perpendicular configurations of the dimers on graphite surfaces. Thirdly, dynamics simulations with a constant temperature scheme are shown to result in a clear picture of the dimer moving to its preferred perpendicular orientation on graphite surfaces at room temperature. Finally, we report on dynamical simulations of a gold dimer under the influence of a fixed STM "tip" composed of a carbon cluster extracted from a diamond lattice. ${ }^{21}$

This paper is organized as follows. Section II gives a description of our computational approach. In Sec. III we present a series of results from PLATO applied to small isolated gold clusters, to gold adatoms at different binding sites, to a dimer in nearly normal or nearly parallel configurations on the graphite surface, to the dynamical simulations of a dimer on the graphite surface, and finally, to dynamical simulations under the effect of tip atoms. These results and discussions are followed by conclusions in Sec. IV. 


\section{COMPUTATIONAL METHODS}

The PLATO (Ref. 20) computational package is used for all calculations. It implements a density functional approach using optimized numerical orbitals extracted from both neutral atoms and positively charged ions. The pseudopotentials for the atomic cores are adopted from the relativistic separable dual-space Gaussian scheme of Hartwigsen, Goedecker and Hutter. ${ }^{22}$ The exchange and correlation functional takes the local density approximation. It was found that including additional orbitals is more effective in increasing structural accuracy for isolated clusters than increasing the cutoff radii for the range of the integrals between centers. In order to obtain the correct triangular structure for $\mathrm{Au}_{3}$, orbitals from $\mathrm{Au}^{4+}$ should be included (resulting in a triple numeric plus polarization basis set), while a set of orbitals including neutral atoms and doubly charged ions is sufficient for accurate calculations involving lighter atoms such as carbon (a double numeric plus polarization basis set). The cutoff radii for gold and carbon atoms are 8 and 7 a.u., respectively. The supercell scheme is followed in all of our calculations and is defined by the number of unit cells in the three primitive vector directions. The supercell for static relaxation contains $3 \times 3$ $\times 2$ primitive cells, including four atomic layers, decorated with vacuum regions above and below. The top two layers along with the deposited particles are allowed to relax. The in-plane size, $3 \times 3$, is sufficient to decouple the interactions between cells for both a single gold atom and a gold dimer. Optimized structures are obtained when the energy converges to at least $8 \times 10^{-4}$ a.u. and the maximum force anywhere in the structure is less than 0.001 a.u. Multiple Bloch states are included with $8 k$ points in the periodic calculation.

Dynamic simulations are carried out using the NoséHoover thermostat at a temperature of $300 \mathrm{~K}$. The time step is set to $1 \mathrm{fs}$ with the maximum number of steps equal to 200. At each time step, the self-consistent calculation of the electronic states is converged. Only the top two layers are kept in these simulations. From the dynamic simulations, clear pictures of the motion of dimers at room temperature and under the effect of STM tip have been observed, although thermodynamic or statistical properties may not be extracted due to the relatively short time scale and the small supercell size.

\section{RESULTS AND DISCUSSIONS}

The effectiveness of our computational scheme was tested by the application of PLATO to small isolated gold clusters. For a gold dimer, PLATO returns a binding energy of 2.86 $\mathrm{eV}$ and a bond length of $2.53 \AA$, in good agreement with the experimental data $(2.61 \mathrm{eV}$ and $2.47 \AA) .{ }^{24} \mathrm{For}_{\mathrm{Au}_{3-7}}$, the PLATO results reproduce all of the lowest energy structures resulting from previous calculations using DFT approaches. ${ }^{11,23}$ Total energy values are collected in Table I and the structures are shown in Fig. 1. These calculations with PLATO include the successful prediction of the ground state triangular structure for $\mathrm{Au}_{3}$, which is found to be 0.05 $\mathrm{eV}$ below the linear form. ${ }^{23}$ The suitability of the PLATO formalism for graphite has been previously reported. ${ }^{12}$ In
TABLE I. Symmetry, total energy $\mathrm{E}_{\mathrm{N}}$ and binding energy per atom of small gold clusters.

\begin{tabular}{lccc}
\hline \hline Size & Symmetry & $\mathrm{E}_{\mathrm{N}}(\mathrm{eV})$ & $\mathrm{E}_{\mathrm{N}} / \mathrm{N}(\mathrm{eV})$ \\
\hline 2 & $\mathrm{D}_{\propto \mathrm{h}}$ & -2.861 & -1.430 \\
3 & Near $\mathrm{D}_{3 \mathrm{~h}}$ & -4.605 & -1.535 \\
& $\mathrm{D}_{\infty \mathrm{h}}$ & -4.553 & -1.518 \\
4 & $\mathrm{D}_{2 \mathrm{~h}}$ & -8.012 & -2.003 \\
5 & $\mathrm{C}_{2 \mathrm{v}}$ & -10.838 & -2.168 \\
& $\mathrm{D}_{3 \mathrm{~h}}$ & -10.123 & -2.024 \\
6 & $\mathrm{D}_{3 \mathrm{~h}}$ & -14.628 & -2.438 \\
& $\mathrm{C}_{5 \mathrm{v}}$ & -13.827 & -2.304 \\
& $\mathrm{C}_{2 \mathrm{v}}$ & -13.615 & -2.269 \\
7 & $\mathrm{D}_{5 \mathrm{~h}}$ & -16.944 & -2.420 \\
& $\mathrm{D}_{6 \mathrm{~h}}$ & -16.739 & -2.391 \\
\hline \hline
\end{tabular}

that study, the surface contraction, defined as the decrease in the distance between the top and second layers, was found to be $0.12 \AA$, a value greater than experimental, $0.05 \AA$. This discrepancy between computational and experimental results was attributed to the known density functional theory difficulty with weak interactions, such as the interlayer attraction in graphite. The success in locating the global minima for the gold clusters and the previous success with the graphite surface provide confidence that PLATO may be applied to the more complex gold adatom-graphite surface problem. ${ }^{12}$

\section{A. Gold adatoms on graphite surfaces}

PLATO was used to determine the preferred binding sites for gold adatoms on graphite surfaces. The results, shown in Table II, indicate that, energetically, $\beta$ binding sites are the most favored and hollow (center of a hexagon) sites are the least favored binding positions. This is in agreement with the STM observation $^{19}$ that a gold adatom was typically located near a $\beta$ site and never observed over a hollow. While the energy differences among the $\alpha, \beta$ and bridge (carboncarbon bond) sites are small, the hollow site is significantly less favored. The binding energies for gold adatoms are systematically larger than those for silver adatoms. The energy landscape along the [110] direction is sketched in Fig. 2, where the top sites $(\alpha$ and $\beta$ ) are local minima and the bridge and hollow points are obtained by constrained relaxation, i.e., adatoms are limited to motion in the normal direction. The energy differences along this direction of the surface are greater for gold than for silver atoms. Decreased mobility was observed for gold, relative to silver adatoms, in STM. ${ }^{19}$ A gold adatom was observed near a $\beta$ site for approximately 10 minutes, compared to only a few seconds for the silver adatom. These experimental results are consistent with the increased energy differences from the calculations. Two other differences between silver and gold adatoms are computationally observed. Gold atoms are negatively charged when bound at any site, whereas silver adatoms are positively charged. Larger surface deformation in the region close to the metal adatom is induced by gold in comparison to silver. 

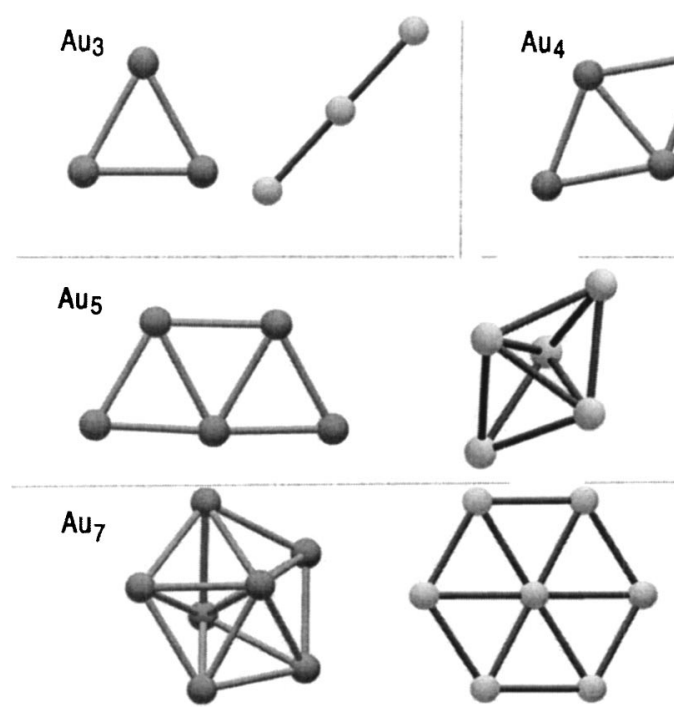

\section{B. Gold dimers on graphite surfaces}

Dimers parallel to the surface. PLATO has also been applied to the adsorption of gold dimers on a graphite surface. Results are collected in Table III for typical binding configurations of a dimer initially oriented parallel to the surface. The center of the gold dimer is set initially above the sites listed in the first column. More detailed descriptions of these initial configurations can be found in a separate publication, ${ }^{12}$ and are only briefly described here. The holeA site means that the dimer is oriented along the diagonal of a hexagon, while for holeB, the dimer spans two opposing bridge sites. There are two cases for the bridge site, one perpendicular (bond 1) and the other parallel (bond 2) to the bond. For the $\alpha$ site case, one gold atom is over a hollow site and the other over a $\beta$ site opposite. A similar situation applies to the $\beta$ site case; one gold is over an $\alpha$ site and the other over a hollow site. The $\beta_{2}$ designation denotes a case with the gold atoms over adjacent $\beta$ sites. The most favored of all parallel cases is the holeA site. This is consistent with the adatom case, since for this structure the two gold atoms are close to one $\alpha$ and one $\beta$ site. When compared to single adatoms, dimers bind at a higher distance above the surface. Since the two atoms encounter different surroundings, they are not necessarily expected to be located at the same height. The height $h$ in Table III is the averaged value for the two atoms. The variable $\eta$ is the angle between the dimer bond

TABLE II. Interactions between a gold adatom and a graphite surface using PLATO with a $3 \times 3 \times 2$ supercell. $\mathrm{E}_{\mathrm{B}}$ is the binding energy, $h$ is the height of the gold atom above the average level of the top layer and $\Delta \mathrm{h}_{\mathrm{c}}$ denotes the (average) height of the closest carbon atom(s) at the binding sites above the rest of other carbon atoms in the top layer.

\begin{tabular}{lcccc}
\hline \hline Site & $-\mathrm{E}_{\mathrm{B}}(\mathrm{eV})$ & $h(\AA)$ & $\Delta \mathrm{h}_{\mathrm{c}}(\AA)$ & Au charge $(e)$ \\
\hline$\alpha$ & 0.662 & 2.457 & 0.148 & -0.160 \\
$\beta$ & 0.674 & 2.468 & 0.276 & -0.165 \\
bridge & 0.654 & 2.475 & 0.162 & -0.143 \\
hollow & 0.512 & 2.621 & 0.016 & -0.093 \\
\hline \hline
\end{tabular}

FIG. 1. Structures of small isolated gold clusters. When more than one isomer is provided, the structure with the darker gold atoms is the most stable conformer. and the surface. Dimers over $\alpha$ and $\beta$ sites feature a significant angle, while dimers for other binding sites are nearly parallel to the surface plane. The bond length, $\mathrm{d}_{\mathrm{Au} 2}$, of the dimers does not significantly vary. This implies that the internal interaction in the dimers is stronger than the dimersurface interaction. The binding energy, $\mathrm{E}_{\mathrm{B}}$, is calculated as the dissociative energy of the dimer from the surface, $\mathrm{E}$ (system)-E(graphite)-2E (gold atom). Also included in the table, is the explicit dimer adsorption energy, $\mathrm{E}_{\mathrm{A}}$, calculated as $\mathrm{E}$ (system)-E(graphite)-E (isolated dimer). The latter quantity assumes that the dimer bond energy on the surface is identical to its value in the gas phase. With such an assumption, we observe that the adsorption energy of the dimer is less than the sum of the binding energy for two single atoms. This is consistent with the previous conclusion that the $\mathrm{Au}-\mathrm{Au}$ interaction is greater than the $\mathrm{Au}-\mathrm{C}$ interaction.

Dimers perpendicular to the surface. We also applied PLATO to $\mathrm{Au}_{2}$ binding with the internuclear axis initially oriented perpendicular, or nearly so, to the graphite surface. Table IV provides the energetics for these dimers. The binding energies, calculated as $\mathrm{E}_{\mathrm{B}}$ or $\mathrm{E}_{\mathrm{A}}$, are systematically larger than those for the parallel cases described above. At all four binding sites, the optimized dimers are nearly perpen-

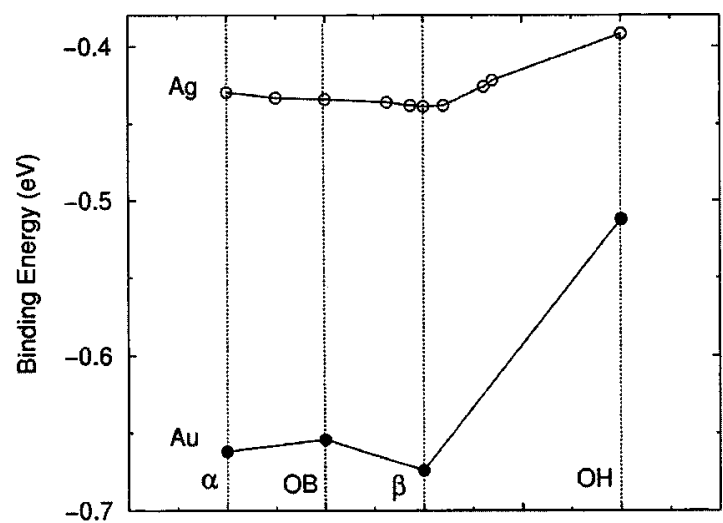

[110]

FIG. 2. Binding energy of gold and silver atoms on graphite surfaces along [110]. $\mathrm{OB}$ is a bridge site and $\mathrm{OH}$ is a hollow. 
TABLE III. The interactions between near parallel gold dimers and graphite surfaces using a $3 \times 3$ in-plane supercell with four atomic layers.

\begin{tabular}{lcccccc}
\hline \hline Site & $-\mathrm{E}_{\mathrm{B}}(\mathrm{eV})$ & $-\mathrm{E}_{\mathrm{A}}(\mathrm{eV})$ & $\mathrm{h}$ & $\mathrm{d}_{\mathrm{Au} 2}$ & $\eta(\mathrm{deg})$. & Charge $($ on Au1/Au2 $)(e)$ \\
\hline$\alpha$ & 3.603 & 0.742 & 2.790 & 2.588 & 15 & $-0.230(-0.190 /-0.040)$ \\
$\beta$ & 3.618 & 0.756 & 2.793 & 2.562 & 16 & $-0.235(-0.194 /-0.041)$ \\
Bond 1 & 3.523 & 0.662 & 2.815 & 2.564 & 0 & $-0.160(-0.080 /-0.080)$ \\
Bond 2 & 3.502 & 0.641 & 2.849 & 2.559 & 0 & $-0.149(-0.075 /-0.074)$ \\
holeA & 3.630 & 0.768 & 2.633 & 2.587 & 0.5 & $-0.220(-0.110 /-0.110)$ \\
holeB & 3.570 & 0.709 & 2.710 & 2.577 & 0.3 & $-0.190(-0.097 /-0.093)$ \\
$\beta_{2}$ & 3.568 & 0.706 & 2.716 & 2.576 & 0.3 & $-0.182(-0.089 /-0.093)$ \\
\hline \hline
\end{tabular}

dicular to the surface as indicated by the small values for the angle $\theta$. This parameter is defined as the angle between the dimer bond and the direction normal to the surface. A dimer over a hollow site is not stable and is expected to quickly relax to a position over one of the other three sites. One may draw the energy landscape of perpendicular dimers along the [110] direction. This landscape will be similar to that shown in Fig. 2 for single adatoms. However, the binding energies per atom indicate that the dimer should be more mobile than the adatom.

There is one reported case in the literature of a dimer found to be more stable when oriented perpendicular rather than parallel to a surface. This was reported for silver dimers on the $\mathrm{MgO}(001)$ surface. ${ }^{25}$ This observation was attributed to the differences in the Pauli repulsion between occupied $\mathrm{Ag}_{2}$ and $\mathrm{MgO}$ orbitals. The repulsive Pauli interaction for a parallel dimer is greater than that for a vertically oriented molecule and the energy released upon binding to the surface is not sufficient to overcome this repulsion. However, the $\mathrm{MgO}(001)$ surface features ionic bonds, while the graphite surface is covalent. Although the $\mathrm{MgO}$ and graphite surfaces are chemically very different, we may draw analogies in the local region of the adsorbed dimer. A silver dimer in the vertical orientation on the $\mathrm{MgO}(001)$ surface sits over a negatively charged oxygen ion, surrounded by positive $\mathrm{Mg}^{2+}$ ions. For vertically oriented $\mathrm{Au}_{2}$ on graphite, the underlying carbon atom is negatively polarized and adjacent carbon atoms are positively charged. For the graphite surface, the charges on the surface atoms are induced by the gold dimer. Our calculations for the silver dimers indicate that the perpendicular orientations have binding energies only $0.06 \mathrm{eV}$ greater than the dimers in the parallel orientation. Since kT at room temperature is approximately $0.03 \mathrm{eV}$, we find no significantly preferred binding orientation for silver dimers. The difference between the binding preferences for gold and silver dimers points to relativistic effects as a factor in the gold preference for perpendicular orientations.

\section{Surface polarization and deformation induced by gold adatoms and dimers}

In Tables II-IV, Mulliken charges for the adatoms and dimers are listed in the last columns. Mulliken charges are calculated as the difference between the atomic number of the atom and the calculated electron density about the atom. The electron density in bonds is partitioned according to standard procedures using the overlap integrals. ${ }^{26}$ The tables indicate that the binding energy is strongly correlated to the charge. The binding energy increases with increasing charge; for example, the correlation coefficient is 0.98 in Table II (adatoms). For the two cases, at $\alpha$ and $\beta$ sites, that involve dimers not oriented perfectly parallel, the upper gold atom exhibits a greater (negative) charge than the atom closest to the surface. Similarly, for vertical configurations, the upper atom has a greater charge than the atoms closer to the surface; the lower atom with less charge binds more tightly to the negatively polarized carbon surface. The charged adatom or dimer induces a charge distribution at the surface. The greater the charge on the adsorbed species, the higher the polarization at the surface, and the stronger the interaction between the adsorbed particle and the surface. Significant Mulliken charges are calculated for the carbon surface atoms. Charge distributions on the carbon atoms nearest to the binding site are sketched in Fig. 3 for a gold adatom at a $\beta$ site. The charge distribution features $\mathrm{C}_{3}$ symmetry with the rotation axis passing through the gold atom and the $\beta$ site. The charge distribution and deformation induced by dimers are shown in Fig. 4(a) for dimers over an $\alpha$ site and (b) for dimers over a bridge site, respectively. The surface polarization for both cases has mirror plane symmetry along the $\alpha-\beta$

TABLE IV. Energetics, configurations and charges for gold dimers perpendicular to a graphite surface using a $3 \times 3$ in-plane supercell with four atomic layers.

\begin{tabular}{lcccccc}
\hline \hline Site & $-\mathrm{E}_{\mathrm{B}}(\mathrm{eV})$ & $-\mathrm{E}_{\mathrm{A}}(\mathrm{eV})$ & $\mathrm{h}_{\text {c.m. }}(\AA)$ & $\mathrm{d}_{\mathrm{Au} 2}(\AA)$ & $\theta(\mathrm{deg})$. & Charge $($ on Au1/Au2 $)(e)$ \\
\hline$\alpha$ & 3.990 & 1.129 & 3.555 & 2.516 & 0.60 & $-0.288(-0.216 /-0.072)$ \\
$\beta$ & 3.999 & 1.137 & 3.542 & 2.513 & 0.33 & $-0.292(-0.222 /-0.070)$ \\
bridge & 4.015 & 1.154 & 3.519 & 2.515 & 0.05 & $-0.286(-0.230 /-0.056)$ \\
hollow & 3.783 & 0.919 & 3.573 & 2.520 & 1.84 & $-0.224(-0.113 /-0.111)$ \\
\hline \hline
\end{tabular}




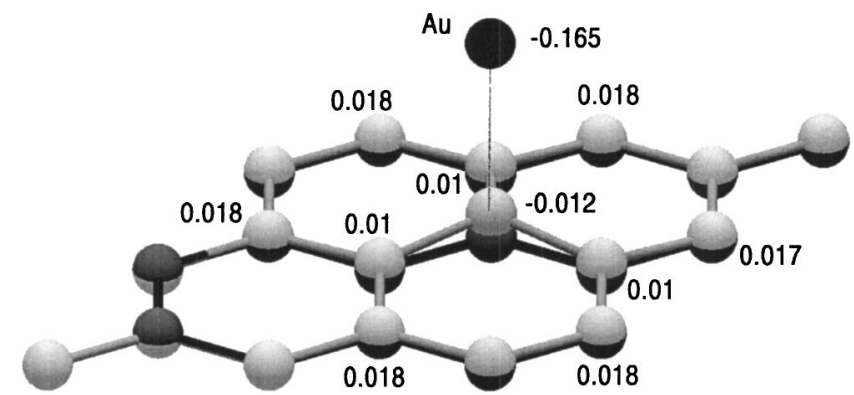

FIG. 3. Surface deformation and surface polarization induced by an $\mathrm{Au}$ atom bound to a $\beta$ site. Darker balls show the top layer with all atoms in a plane, before it is deformed. White balls show the deformed surface and the black ball denotes the gold atom. Similar conventions are used for Fig. 4.

axis. The charges on the carbon atoms nearest to the binding site are positive, forming dipoles from the surface to the deposited particles.

The deformation of the surface layer is demonstrated clearly in the surface polarization figures (Figs. 3 and 4). In the figures, darker balls are artificially drawn in the original surface plane in order to provide a graphical representation of the deformation in the top layer (in the direction normal to the surface). For both adatoms and dimers, the surface deformation is characterized by an elevation of the carbon atoms
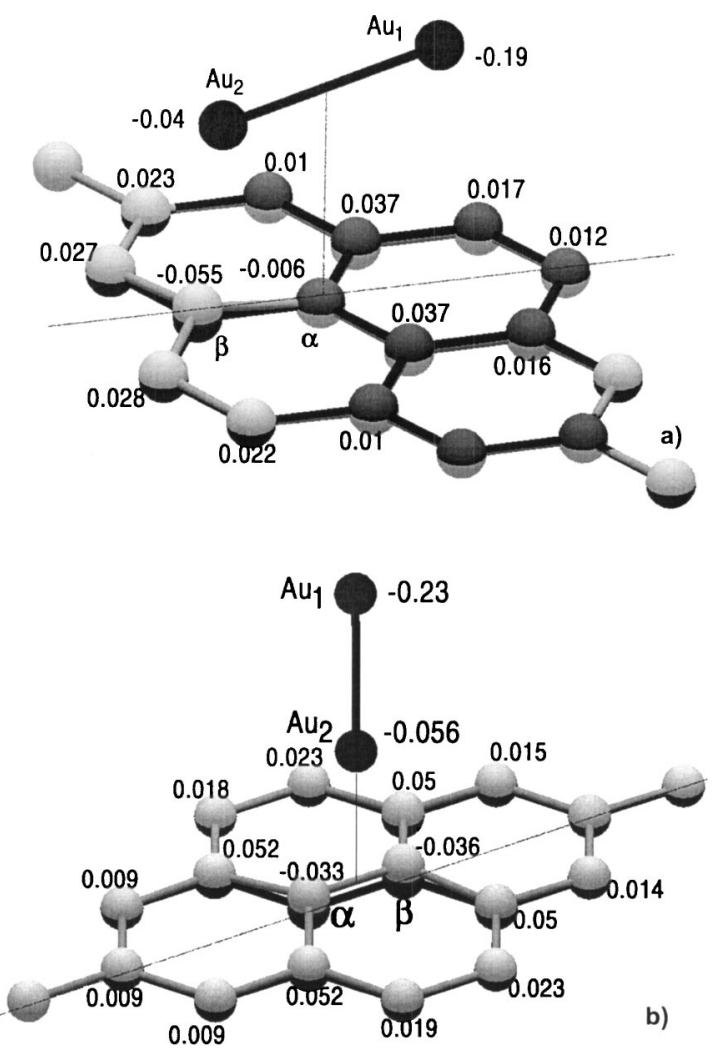

FIG. 4. Surface deformation and surface polarization induced by (a) a gold dimer (black dumbbell) over an $\alpha$ site. Dot-dashed line denotes the symmetry plane of the surface polarization. (b) A gold dimer perpendicular to the surface (black dumbbell) over a bridge site.
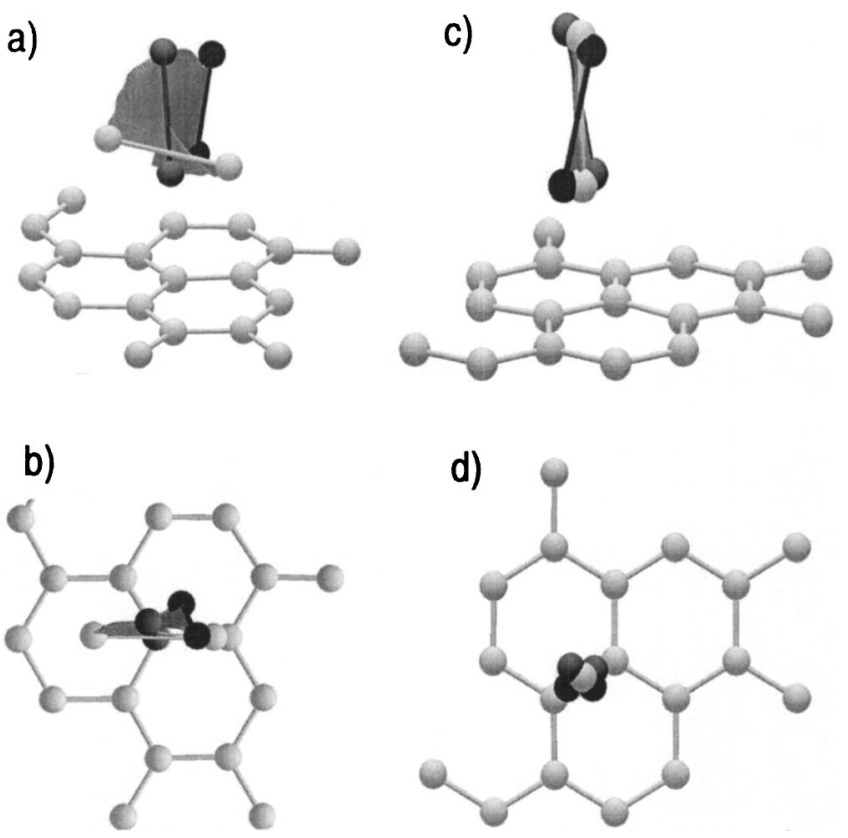

FIG. 5. Dynamic simulations of dimers on graphite surfaces using the Nosé-Hoover thermostat at $300 \mathrm{~K} ; 3 \times 3 \times 1$ primitive cells. Only the top atomic layer is shown. (a) Dimer is initially in a near parallel orientation over a $\beta$ site and equilibrates after $0.1 \mathrm{ps}$ to a near perpendicular orientation over a bridge site. (b) Top view of (a). (c) Dimer is initially in a perpendicular configuration over a bridge site and remains nearly perpendicular. (d) Top view of (c).

closest to the binding site. Surface deformation is confined to the top layer; the second layer of the graphite is seldom disturbed at all.

\section{Dynamical simulation of $\mathrm{Au}_{2}$ on a graphite surface}

Dynamic simulations of gold dimers with two initial configurations at $300 \mathrm{~K}$ on a graphite surface were completed and the results are shown in Fig. 5. A Nosé-Hoover thermostat was used in order to overcome potential energy barriers in the approach to the global minimum. In one simulation, shown in Figs. 5(a) and 5(b), the dimer begins from a near parallel configuration (white dumbbell) over a $\beta$ site and is seen to be equilibrated after $0.1 \mathrm{ps}$ in a near perpendicular orientation over a bridge site (darker dumbbell). The angle $\theta$ changes from $75^{\circ}$ to nearly zero over this time period. After equilibration, the simulation was continued for another 0.1 ps. During this period, the dimer is observed to move about a vector normal to the surface. The angle $\theta$ during this time is confined to a value of less than $5^{\circ}$. The movement of the dimer bond sweeps out a three dimensional surface during the simulation. From the top view shown in Fig. 5(b), one observes from this surface that the center of mass of the dimer does not significantly change during the simulation.

In the second simulation, the dimer begins from a perpendicular orientation over a bridge site and remains nearly perpendicular with only slight variations in the center of mass during the $0.1 \mathrm{ps}$ simulation. The side and top views of these changes during the simulation are shown in Fig. 5(c) and $5(\mathrm{~d})$, respectively. The angle $\theta$ remains within a $0-5$ degree 
a)
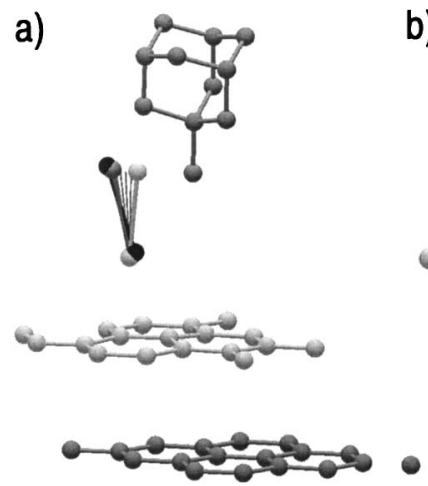

c)

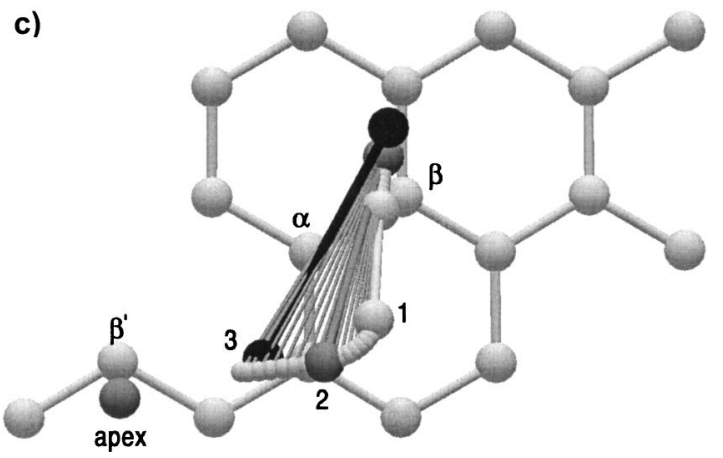

FIG. 6. (a) Effect of STM tip at position II. A fixed diamond tip of 11 atoms is above the two layer surface. Also shown are three instantaneous positions of a dimer: initial (white dumbbell), midsimulation transient (darker dumbbell), and equilibrated (black balls). Other instantaneous positions are shown by the position of the Au-Au bond only. (b) The top view of (a), but for clarity only the apex atom of the tip and the top layer of the surface are included. (c) Effect of a STM tip with apex over a $\beta$ site (tip position IV).

range during this $0.1 \mathrm{ps}$ period. The simulation results are consistent with the optimizations reported in Tables III and IV. The most stable dimer orientation is perpendicular to the graphite surface. Dimers in (near) parallel configurations tend to move to a perpendicular orientation with little apparent energy barrier. A simulation (not shown) of a perpendicularly oriented dimer over a hollow site found this position to be unstable and the dimer moved to one of the stable binding sites, through a relaxation process.

\section{E. Effect of STM tips on gold dimers}

In an attempt to investigate the effect of an STM tip on an adsorbed dimer, a cluster of 11 carbon atoms extracted from a diamond lattice is used to simulate an STM tip as shown in Figs. 6 and 7. The simulation is begun with the dimer-surface system in the equilibrated geometry shown in Fig. 5(c). During all simulations, the STM tip is maintained perpendicular to the surface at a distance equal to the height of the upper gold atom from the surface as defined in the simulation described by Fig. 5(c). Dynamic simulations are completed for

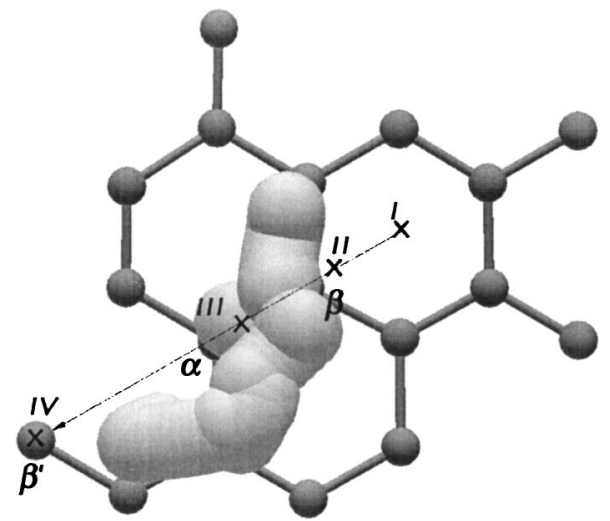

FIG. 7. Top view of the superposition of the instantaneous positions of the dimer from dynamical simulations with all four fixed tip positions. Tip positions used in the simulations are indicated by an $x$ and numbered in the order that they are encountered.

four fixed tip positions in the order shown in Fig. 7. Once equilibration is reached at a tip position, the equilibrated geometry is used as the initial condition for the simulation at the next tip location. At the third position, when the tip is directly over the dimer, the tip is raised $2.3 \AA$ in the direction normal to the surface. This tip movement, corresponding to the tip motion in STM experiments, is arbitrarily chosen and represents the distance between the lower gold atom and the surface at the start of the series of simulations (Fig. 5).

Figure 6(a) shows the simulation results when the tip is located between $\beta$ and hollow sites. Three instantaneous configurations of the dimer are shown: the initial (white dumbbell), the mid-simulation transient (darker dumbbell), and the equilibrated position (black dumbbell). Other instantaneous positions are indicated by the Au-Au bond only. Figure 6(b) provides the top view of (a), with just the apex atom of the tip and the top layer of the surface shown for clarity.

Figure 6(c) shows the dynamics simulation picture with the tip at the fourth position, over a $\beta$ site. Again, three instantaneous configurations as described for Fig. 6(a) are shown. The upper gold atom moves over a much larger region of the surface than does the lower atom. Several instantaneous, non-equilibrated states are shown as the smaller white balls for the upper gold atom. The trace of this atom is bent towards the surface, relative to the initial perpendicular orientation. The upper atom is deflected toward the underlying carbon-carbon bond by the tip. The dimer does not follow the tip indefinitely, but returns to the preferred vertical orientation after the tip passes. This was verified by using the geometry shown in Fig. 6(c) and moving the tip past the dimer. A final simulation was completed using this starting point and the dimer was observed to equilibrate to a vertical position during the 0.1 -ps simulation. Figure 7 provides the top view of the superposition of instantaneous positions of the dimer from all of the dynamics with the four fixed tip positions. The apex positions are indicated by an $x$. The angle $\theta$ varies between $5^{\circ}$ and $55^{\circ}$.

The overall picture of the dimer motion in Fig. 7 may be loosely compared with the image obtained in an STM experiment. ${ }^{19}$ In that STM experiment, the gold dimer bondlength was observed to be longer than that reported for 
the isolated gold dimer. In such a comparison, we must keep in mind that a direct comparison of STM images to theory requires estimates of the tunneling current that may only be obtained by integrating the density of states at the surface. The calculations reported here were not intended to directly provide a simulated STM image. There are several possible reasons for the change in $\mathrm{Au}-\mathrm{Au}$ bondlength observed in the STM experiment. The Au-C interaction may be greater than that for $\mathrm{Au}-\mathrm{Au}$, so that the gold atoms reach a potential well by a lengthening of the bond to bind to more favorable surface sites. Alternatively, the gold-carbon interaction may be weak so that the dimer has a high surface mobility and the apparently increased bondlength is a reflection of slight motion on the surface. Finally, the STM tip may interact with the dimer and cause movement of the adsorbed species as it passes over. Our dynamical simulations suggest that there may be an observable impact of the tip on the gold dimer position during STM scanning. The simulations indicate that tip attracts the vertically positioned dimer as it approaches from a distance. As the tip moves closer to the upper gold atom of the dimer, the dimer is repelled, but tends to remain above or along a carbon-carbon bond. Finally, the upper gold atom returns to its equilibrium position as the tip passes by. In contrast, the motion of the lower gold atom is much more limited and confined to a small region over the initial bridgesite location.

\section{CONCLUSIONS}

In this paper, accurate calculations using a density functional approach are reported for gold adatoms and dimers on relaxed graphite surfaces. Results for adatoms on graphite surfaces are in qualitative agreement with STM observations. Based upon the energetics of the adatoms, $\beta$ sites are favored by single gold atoms and hollow sites are not stable. However, several types of binding sites have comparable energies and are essentially indistinguishable based on this criterion. The energy landscape indicates that a gold adatom is not as mobile as a silver adatom, also in agreement with STM experiments. Static calculations and dynamical simulations for a dimer on the graphite surface indicate that the dimer prefers to stand vertically on the surface. This phenomenon has not been previously reported for dimers on covalent surfaces such as graphite.

We also observed that adatoms and dimers induce surface polarization, as well as surface deformation. These results indicate that surfaces or deposited particles and STM tips will interact both mechanically and electronically. An asymmetry of STM images with respect to $\beta$ site carbons in graphite has been observed by several groups. ${ }^{18,19}$ Whangbo et al. $^{27}$ concluded from experimental work that this feature of STM images derived from both mechanic and electronic effects. Our findings of surface polarization and deformation induced by deposited particles support this theory.

\section{ACKNOWLEDGMENT}

The financial assistance of the NATO Division of Scientific and Environmental Affairs through a Collaborative Linkage Grant, PST.CLG.978441, is gratefully acknowledged.
${ }^{1}$ K. Bromann, C. Félix, H. Brune, W. Harbich, R. Monot, J. Buttet, and K. Kern, Science 274, 956 (1996).

${ }^{2}$ C. Bréchignac, Ph. Cahuzac, F. Carlier, C. Colliex, J. Leroux, A. Masson, B. Yoon, and U. Landman, Phys. Rev. Lett. 88, 196103 (2002).

${ }^{3}$ P. J. Feibelman, Phys. Rev. Lett. 58, 2766 (1987).

${ }^{4}$ M.-H. Schaffner, J.-F. Jeanneret, F. Patthey, and W.-D. Schneider, J. Phys. D 31, 3177 (1998).

${ }^{5}$ L. Motte, E. Lacaze, M. Maillard, and M. P. Pileni, Langmuir 16, 3803 (2000).

${ }^{6}$ D. M. Duffy and J. A. Blackman, Surf. Sci. 415, 1016 (1998).

${ }^{7}$ I. Moullet, Surf. Sci. 331-333, 697 (1995).

${ }^{8}$ G. Bravo-Pérez, I. L. Garzón, and O. Novaro, J. Mol. Struct. 493, 225 (1999).

${ }^{9}$ H. Grünbeck and W. Andreoni, Chem. Phys. 262, 1 (2000).

${ }^{10}$ H. Häkkinen and U. Landman, Phys. Rev. B 62, 2287 (2000).

${ }^{11}$ J. Wang, G. Wang, and J. Zhao, Phys. Rev. B 66, 035418 (2002);

B. Delley, J. Chem. Phys. 92, 508 (1990); DMol is distributed by Accelrys.

${ }^{12}$ G. M. Wang, J. J. BelBruno, S. D. Kenny, and R. Smith, Surf. Sci. 541, 91 (2003).

${ }^{13}$ C. F. Sanz-Navarro, R. Smith, D. J. Kenny, S. Pratontep, and R. E. Palmer, Phys. Rev. B 65, 165420 (2002).
${ }^{14}$ S. J. Carroll, S. Pratontep, M. Streun, R. E. Palmer, S. Hobday, and R. Smith, J. Chem. Phys. 113, 7723 (2000).

${ }^{15}$ X. Bouju, C. Joachim, and C. Girard, Phys. Rev. B 50, 7893 (1994).

${ }^{16}$ H. Rafii-Tabar, H. Kamiyama, and M. Cross, Surf. Sci. 385, 187 (1997).

${ }^{17}$ D. Brenner, Phys. Rev. B 42, 9458 (1990).

${ }^{18}$ F. Atamny, O. Spillecke, and R. Schlögl, Phys. Chem. Chem. Phys. 1, 4113 (1999).

${ }^{19}$ E. Ganz, K. Sattler, and J. Clarke, Surf. Sci. 219, 33 (1989).

${ }^{20}$ S. D. Kenny, A. P. Horsfield, and H. Fujitani, Phys. Rev. B 62, 4899 (2000).

${ }^{21}$ V. T. Binh and J. Marien, Surf. Sci. 202, L539 (1988).

${ }^{22}$ C. Hartwigsen, S. Goedecker, and J. Hutter, Phys. Rev. B 58, 3641 (1998).

${ }^{23}$ R. Guo, K. Balasubramanian, X. Wang, and L. Andrews, J. Chem. Phys. 117, 1614 (2002).

${ }^{24}$ M. D. Morse, Chem. Rev. (Washington, D.C.) 86, 1049 (1986).

${ }^{25}$ A. M. Ferrari, C. Xiao, K. M. Neyman, G. Pacchiono, and N. Rüsch, Phys. Chem. Chem. Phys. 1, 4655 (1999).

${ }^{26}$ W. J. Hehre, L. Radom, P. v. R. Schleyer, and J. A. Pople, $A b$ Initio Molecular Orbital Theory (Wiley New York, 1986).

${ }^{27}$ M.-H. Whangbo, W. Liang, J. Ren, S. N. Magonov, and A. Wawkuschewski, J. Phys. Chem. 98, 7602 (1994). 